\title{
Short-Period Oscillations of Earth Rotation
}

\author{
B. Kołaczek, W. Kosek \\ Space Research Centre, PAS Warsaw, Poland
}

H. Schuh

Deutsches Geodätisches Forschungsinstitut, DGFI München, Germany

\begin{abstract}
.
Sub-seasonal variations and especially sub-seasonal oscillations with periods of about $120,60,50,40$ days in polar motion and of about 120 , 60-90, and 50 days in LOD are presented. Variations of amplitudes of these sub-seasonal oscillations of polar motion are shown. Maxima of these amplitudes are of the order of 2-4 mas. These oscillations are elliptical ones. The correlation coefficients between geodetic and atmospheric excitation functions in this range of the spectrum are variable and have annual variations. Maxima of correlation coefficients are of the order of 0.6-0.8.

Modern geodetic VLBI experiments provide very accurate results in polar motion and UT1-UTC with a temporal resolution of 3-7 minutes. Several irregular, quasi-periodic variations were found. In many UT1-UTC data sets, oscillations with periods around 8 hours and between 5 and 7 hours can be seen.
\end{abstract}

\section{Introduction}

Improvement of the accuracy and temporal resolution of the Earth rotation parameters (ERP) achieved by the application of satellite (SLR, GPS) and VLBI methods in the last two decades have enabled detection and study of sub-seasonal, diurnal, sub-diurnal and even irregular variations of ERP (IERS, 1988-1998). At present the accuracy of polar motion and length of day (LOD) determinations is of the order of 0.1 mas and $0.01 \mathrm{~ms}$, respectively (see IERS Annual Report for 1998).

The new methods of spectral analyses and filtration, for instance the Fourier Transform Band Pass Filter (FTBPF), the Wavelet Transform, the Kalman filter have been developed and applied to these studies improving analyses of variations of ERP (Brzeziński, 1995; Gambis, 1992; Kosek, 1987, 1995; Kosek et al., 1995a,b, 1998a; Morabito et al., 1988; Petrov et al., 1996; Popiński et al., 1994, 1995 a, b; Schmitz-Hübsch and Schuh, 1999; Titov, 1999).

The sub-seasonal variations of polar motion and LOD containing oscillations with periods shorter than half a year were not only detected (Eubanks et al., 1988; Feissel et al., 1980; Hide et al., 1991; Kołaczek et al., 1987; Kołaczek, 
1995; Kosek 1987, 1993; Nothnagel et al., 1992; Schuh 1986, 1988) but their time variations have been found (Kołaczek 1992, 1993; Kolaczek et al., 1993; Kosek et al., 1995, 1998b, 1999a,b; Nastula, 1992; 1995; Popiński et al., 1994; Schmitz-Hübsch and Schuh, 1999).

Correlations between sub-seasonal variations of Earth rotation and subseasonal variation's of Effective Atmospheric Angular Momentum functions were determined and studied. Influences of $\mathrm{El} \mathrm{Niño} \mathrm{events} \mathrm{on} \mathrm{this} \mathrm{correlation} \mathrm{have}$ been established (Brzeziński, 1992; Chao, 1989, 1993; Dickey 1993; Dickey et al., 1991, 1992; Eubanks et al., 1985; Freedman et al., 1994; Gambis, 1992; Hide et al., 1980, 1991; Eubanks, 1993; Kołaczek et al., 1999; Kosek et al., 1995b; Kuehne et al., 1993; Nastula, 1995; Nastula et al. 1997; Rosen and Salstein, 1983; Schmitz-Hübsch and Schuh, 1999; Schuh 1999).

Recently modern geodetic VLBI experiments provide very accurate results of polar motion and UT1-UTC with a temporal resolution of 3-7 minutes. Wavelet transformations of the time series obtained by VLBI allow us to detect several irregular quasi-periodic variations. In many UT1-UTC data sets oscillations with periods around several hours were found (Schuh and Titov, 1999). Using the Least-Squares Collocation Method (LSCM) also high-resolution polar motion series were computed and analysed. Oscillations with periods around several hours were also found in these series (Schuh and Titov, 1999).

In the paper the computed sub-seasonal variations of polar motion and LOD, especially their most energetic oscillations with periods of about 120 , $60-90,60,50,40$ days, are presented and discussed. Results of recent VLBI determinations of quasi-periodic subdaily oscillations of UT1-UTC and polar motion are shown.

\section{Sub-seasonal variations of ERP}

Sub-seasonal variations of ERP are still quite energetic in comparison with the present accuracy of ERP determinations and contain important information about geophysical influences on ERP variations.

Sub-seasonal variations of polar motion filtered from the IERS C04 pole coordinate data using the Butterworth high pass filter (HPF) (Otnes et al., 1972) with a cutoff period of 150 days have variable amplitudes reaching maxima of about 10 mas (Fig. 1). Perturbations of polhodia caused by these short-period variations are shown in Figure 2. Several energetic oscillations are visible in the spectra of these short-period variations computed by the FTBPF (Kosek, 1995; Popiński et al., 1995b). The prograde oscillations with periods of about 120,60, 50,40 days are the most energetic ones (Fig. 3).

These oscillations were filtered by the FTBPF (Kosek, 1995) and are shown in Figure 4. Amplitudes of these oscillations vary in time and are modulated with a period of about 2-4 years. These elliptical oscillations are presented in Figure 5, where polhodia of these oscillations are drawn for some years for example. 120,60 and 40 day oscillations can be of seasonal origin.

Time variable spectra of the short-period variations of the IERS C04 polar motion filtered by the Butterworth HPF with cutoff period of 90 and 150 days computed by the FTBPF are shown in Figure 6 . We can easily notice amplitude variations of these oscillations and not very stable periods. These oscillations 


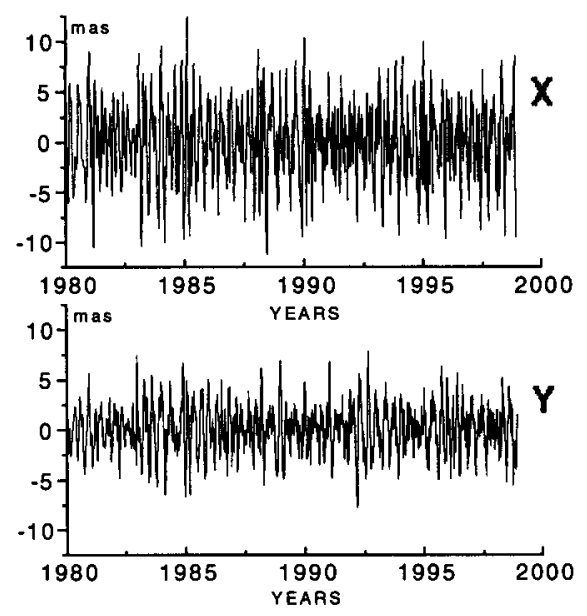

Figure 1. Short-period oscillations of polar motion IERS C04 filtered by the Butterworth HPF with 150-day cutoff period.

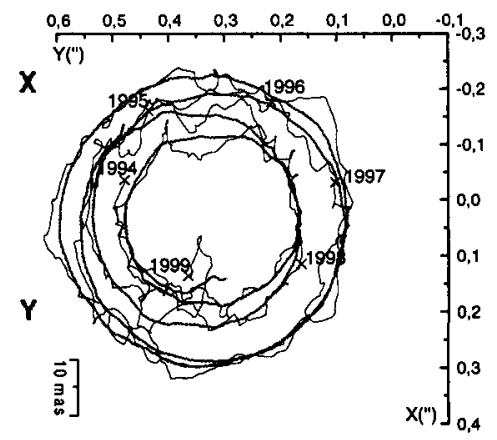

Figure 2. Polar motion IERS C04 determined in 1994-1999. The thin line represents the short-period variations with periods less than 150 days increased by 5 times. The scale of their amplitudes is given in the lower left corner. 


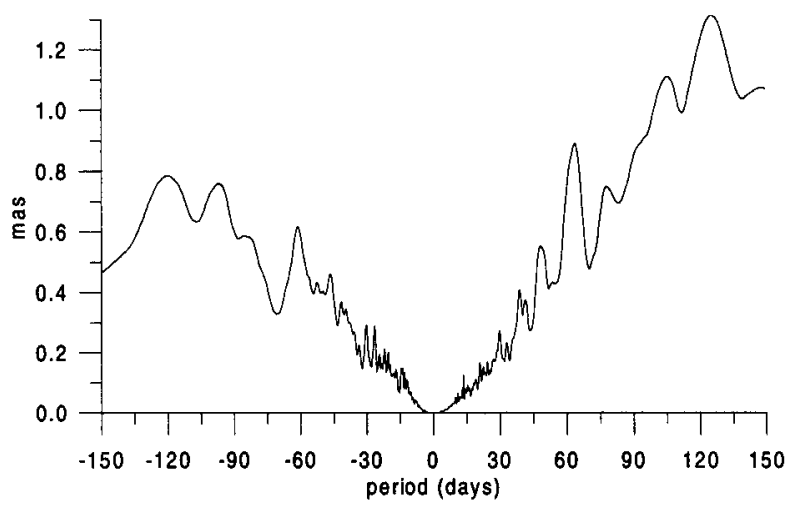

Figure 3. The FTBPF amplitude spectra $(\lambda=0.001)$ of $x$-iy IERS C04 pole coordinate data in 1984.0-1999.0 filtered by the Butterworth HPF with 150-day cutoff period (Kosek et al. 1998b).
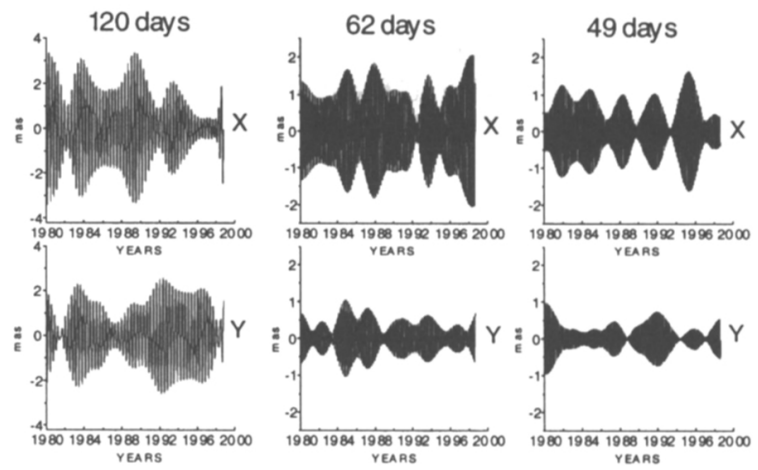

Figure 4. 120, 62 and 49 days oscillations filtered by the FTBPF $(\lambda=0.001)$ from the IERS C04 pole coordinates data.

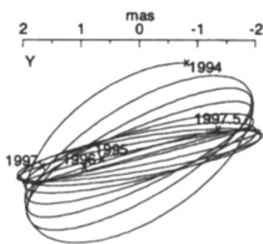

120 days

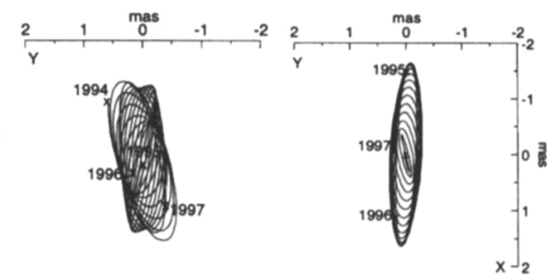

62 days

49 days

Figure 5. The polhodes of 120,62 and 49 days oscillations filtered by the FTBPF $(\lambda=0.001)$ from the IERS C04 pole coordinate data. 

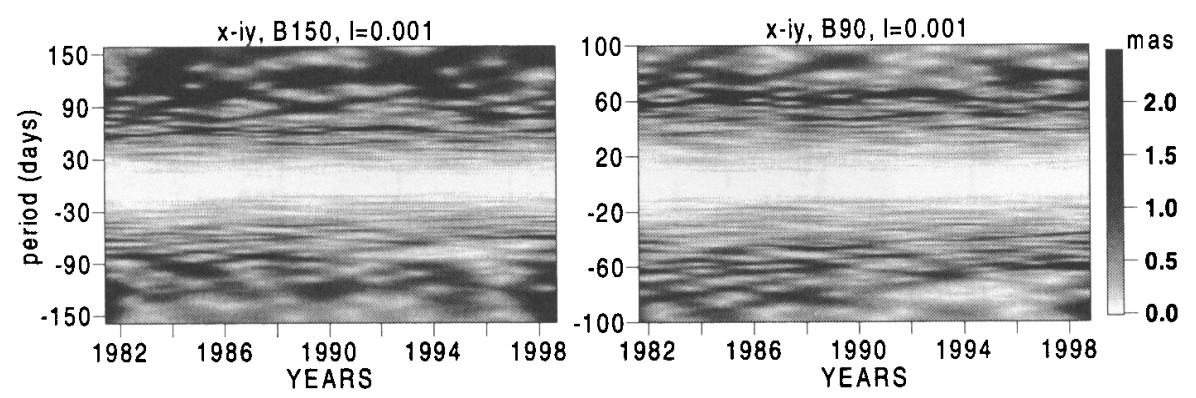

Figure 6. The time variable FTBPF amplitude spectra $(\lambda=0.001)$ of $\mathrm{x}$-iy IERS C04 pole coordinates data filtered by the Butterworth HPF with 150 and 90 day cutoff periods (Kosek et al., 1998b).

are not very strong. Thus they can be easily disturbed by different geophysical phenomena.

Sub-seasonal variations of polar motion are correlated with short-period variations of equatorial components of Atmospheric Angular Momentum (AAM). In the spectra of AAM there are short-period oscillations with periods of about $120,90,60$, and 50 days, similar to those in polar motion (Kosek et al., 1995). The running correlation coefficients between short-period variations of the atmospheric equatorial components (NCEP/NCAR w+p+ib) and geodetic IERS C04 excitation function of polar motion were computed (Fig. 7) (Kosek et al., $1998 \mathrm{~b})$. The correlation coefficients are time variable and have seasonal variations. Their maxima reach values of $0.6-0.7$.

LOD sub-seasonal variations obtained by filtering the IERS C04 data using the Butterworth HPF with cutoff period of 150 days have amplitudes reaching maxima of $0.5 \mathrm{~ms}$. Before the filtering, the influence of solid Earth tides was removed according to the IERS Conventions (IERS, 1996).

The computed amplitude spectrum and time variable spectrum of the LOD sub-seasonal oscillations shows that the most energetic oscillations are the oscillations with periods of about $120,60-90,50$ and 40 days (Fig. 8). The sub-seasonal variations of LOD are highly correlated with the variations of the axial component of the Atmospheric Angular Momentum and the correlation coefficients are close to 1 for oscillations with periods longer than 20 days (Kosek et al., 1999a). This correlation was studied by many authors (Dickey, 1989; Eubanks 1993; Hide et al., 1991). As in the case of polar motion the oscillations of LOD with periods of $120,60-90$ and 40 days can be of seasonal origin. The 50 day oscillation in LOD is considered to be connected with stratospheric winds.

\section{Daily and sub-daily variations of Earth rotation parameters ob- served by VLBI}

Today, a very high temporal resolution of the Earth rotation parameters can be achieved by VLBI using the least-squares collocation method (LSCM). It allows 

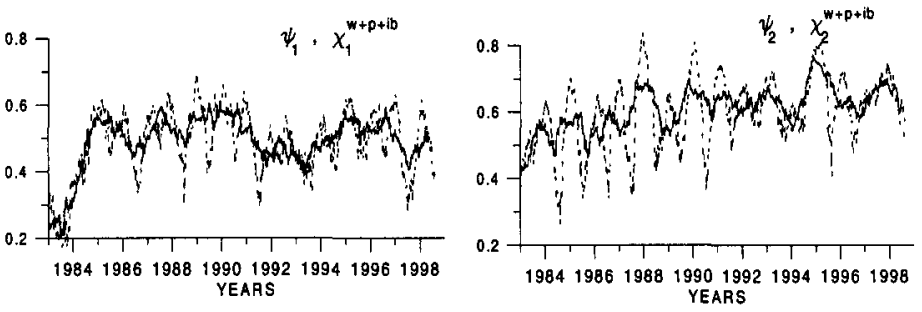

Figure 7. The running correlation coefficient between the atmospheric (NCEP/NCAR ( $w+p+i b))$ and geodetic IERS C04 excitation functions filtered by the Butterworth HPF with a 150-day cutoff period, (thin and dotted lines denote 1 year and 0.5 year means, respectively) (Kosek et al., 1998b).
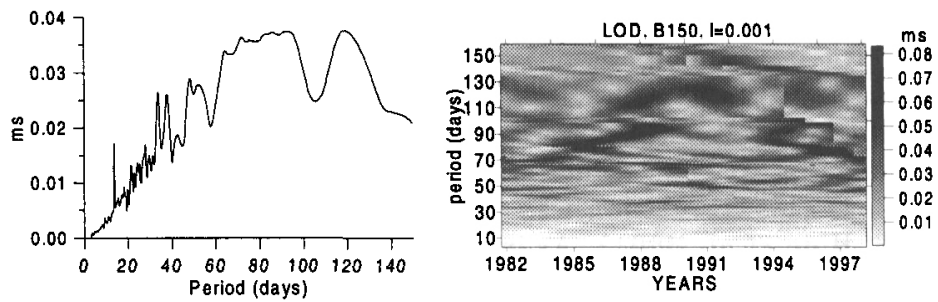

Figure 8. Amplitude spectrum and time variable amplitude spectrum $(\lambda=0.001)$ of LOD IERS C04 data filtered by the Butterworth HPF with 150-day cutoff period (Kosek et al., 1998b). 
Figure 9. UT1-UTC parameters with high temporal resolution determined during five days of the VLBI session CONT which started on September 30, 1996 (ocean tidal terms were removed from the observations using the IERS Conventions (IERS, 1996) model).

us to estimate the Earth rotation parameters every 3-7 minutes (Titov, 1999). This paragraph concentrates on short-period variations of the Earth rotation parameters which can be seen when analysing the VLBI results. More than 300 VLBI experiments (NEOS-A from 1993 till 1998, CONT'96, CORE) each of them covering $24 \mathrm{~h}$ or $120 \mathrm{~h}$ were analysed by the OCCAM 3.4 VLBI software (Titov et al., 1997). The high-resolution UT1-UTC data series and polar motion data series obtained by the VLBI solutions were analysed with respect to periodic variations. First, the main diurnal and semidiurnal variations due to the ocean tides were removed using the IERS Conventions (IERS, 1996) correction model. As an example Figure 9 shows UT1-UTC parameters determined during five days of the VLBI campaign CONT in 1996.

The residuals, i.e. after correction of oceanic tidal terms, offer the opportunity to search for even smaller variations in the rotation of the Earth. Those are supposed to be e.g. due to resonances with modes of the Earth, the oceans or the atmosphere or due to excitation by earthquakes or by strong typhoons. The wavelet transformation was applied to search for transient and irregular variations. Although ocean tidal influences had been already corrected, many of the wavelet spectra of the UT1 series show residual energy in the diurnal and semidiurnal period range. Additionally, most of the data sets revealed irregular quasi-periodic fluctuations which are non-diurnal and non-semidiurnal. Very often (but not always) periods at 20 hours and 40 hours, around 8 hours, between 5 and 7 hours and even down to 2 to 3 hours were found. To give an example of that, the wavelet spectrum for the CONT'96 UT1-UTC series shown in Figure 9 is plotted in Figure 10 for periods below 36 hours. Besides the residual diurnal and semidiurnal bands mentioned above, irregular periodic variations can be seen at $20 \mathrm{~h}, 8 \mathrm{~h}, 5 \mathrm{~h}$ and $2 \mathrm{~h}-3 \mathrm{~h}$ that are above the error level of the individual UT1-UTC parameters of about 10 microseconds. The VLBI so- 
Kolaczek et al.

Wavelet spectrum of UT1-UTC observed by VLBI

(30.09.-05.10.96)

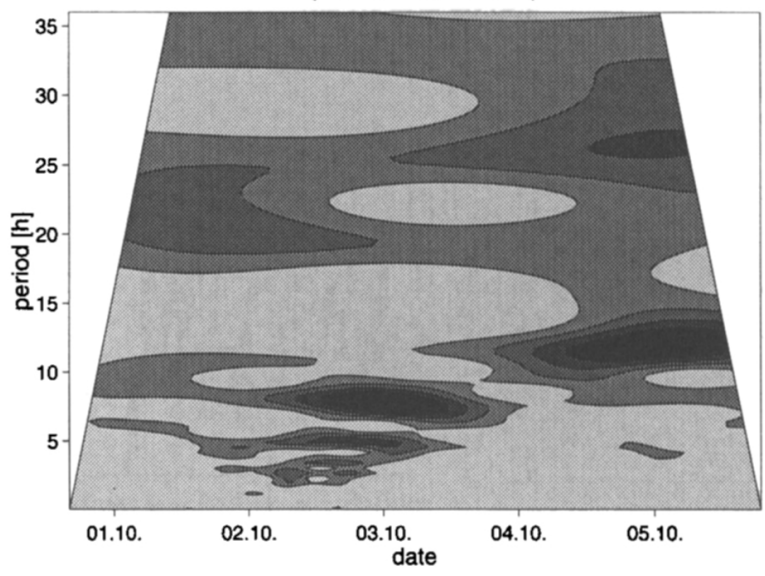

Figure 10. Wavelet spectrum for periods below 36 hours of the highresolution UT1-UTC series shown in Figure 9.

lutions using the LSCM yielded also high-resolution polar motion series. These were analysed as complex values by the wavelet transformation. The wavelet spectra revealed irregular prograde and retrograde short-period fluctuations in polar motion, again very often around 8 hours and even with shorter periods, e.g. at $5-7$ hours.

\section{Conclusions}

In the paper the character of variations of the most energetic sub-seasonal oscillations of polar motion with periods of $120,60,50$ days and of LOD with periods of $120,60-90,50$ and 40 days is described. They have variable amplitudes modulated with a period of 2-4 years. The sub-seasonal variations of polar motion and LOD are correlated with sub-seasonal variations of AAM. The running correlation coefficients are variable with maxima ranging between 0.6 and 0.8 . They have seasonal variations.

High resolution Earth rotation data observed by VLBI have begun to reveal hitherto unseen phenomena. Irregular, quasi-periodic variations can be seen in the wavelet spectra besides the well-known diurnal and semidiurnal periods. Possible excitation mechanisms for the observed variations of the Earth rotation parameters such as terdiurnal tides, the free oscillations of the oceans $(10 \mathrm{~h}, 20 \mathrm{~h}$, $40 \mathrm{~h}, 50 \mathrm{~h})$, atmospheric modes and resonances with free oscillations of the Earth (e.g. the Slichter mode) are under discussion. A triggering by strong earthquakes or by a typhoon should also be considered. Although the tiny variations which could be seen are above the error level of the observations careful tests have to be done to check their significance. In particular, the correlation with tropospheric refraction at the observing stations is going to be investigated.

Acknowledgments. This paper was supported by the grant of the Polish Committee of Scientific Research No 52/T12/97/13 under the leadership of 
Prof. B. Kołaczek and the Polish Academy of Sciences / the National Science Foundation Project "Regional Atmospheric Forcing for Polar Motion" directed by Drs B. Kolaczek and D.A. Salstein from the Atmospheric and Environmental Research Incorporated, Cambridge, Massachusetts. The highly-resolved Earth rotation data observed by VLBI were obtained from Dr. O. Titov, Univ. St. Petersburg.

\section{References}

Brzeziński A., 1992. Polar motion excitation by variations of the Effective Atmospheric Angular Momentum functions. Considerations Concerning Deconvolution Problem, MN, 17, 3-20.

Brzeziński A., 1995. "On the Interpretation of Maxium Entropy Power Spectrum and Cross Power Spectrum in Earth Rotation Investigations." Manuscripta Geodetica, 20, 248-264.

Chao B.F., 1989. "Length of day variations caused by El Niño Southern oscillation and quasi-biennial oscillation." Science, 243, 923-925.

Chao B.F., 1993. "Excitation of Earth's polar motion by Atmospheric Angular Momentum variations, 1980-1990." Geophysical Research Letters, 20, 235-256.

Dickey J.O., 1989. Axial Rate of Spin of the Earth, in the Interdisciplinary Role of Space Geodesy (Mueller and Zerbini eds.) Erice, Italy, July 23-29, 1988, 23-30.

Dickey J.O., 1993. Atmospheric excitation of the Earth's rotation: Progress and prospects via space geodesy, AGU Monograph, Contributions of space geodesy to geodynamics: Earth Dynamics, Smith and Turcotte eds., Geodynamics Series, 24, 55-70.

Dickey J.O., Ghil M., Marcus L., 1991. "Extratropical aspects of the 40-50 day oscillation in Length of Day and Atmospheric Angular Momentum." $J G R$, 96, 22, 643-658.

Dickey J.O., Marcus S.L., Eubanks T.M., and Hide R., 1993. Climate Studies via Space Geodesy: Relationships between ENSO and and Interannual Length of Day Variations. In Interactions Between Global Climate Subsystems: The Legacy of Hann, Geophys. Monograph, Ser., 75, edited by G.A. McBean and M. Hantel, AGU, Washington D.C. 141-155.

Eubanks T.M., Steppe J.A., Dickey J.O., Collehan P.S., 1985. "A spectral analysis of the Earth's Angular Momentum budget." JGR, 90, 538.55404 .

Eubanks T.M., Steppe J.A., Dickey J.O., Rosen R.D., Salstein D.A., 1988. "Causes of rapid motion of the Earth Pole." Nature, 334, No. 6178, 115-119.

Eubanks T.M., 1993. "Variations in the orientation of the Earth," AGU Monograph, Contributions of Space Geodesy to Geodynamics: Earth Dynamics, Smith and Turcotte eds. Geodynamics Series, 24, 1-54.

Feissel M., Gambis D., 1980. "La mise en evidence de variations rapides de la duree du jour." C.R. Acad. Sc., Paris, 291, 271-273. 
Freedman A.P., Steppe J.A., Dickey J.O., Eubanks T.M. and Sung L.Y., 1994. "The short-term prediction of universal time and length of day using Atmospheric Angular Momentum." JGR, 99, 6981-6996.

Gambis D., 1992. "Wavelet transform analysis of the length of the day and El Niño/Southern oscillation variations at interseasonal and interannual time scale." Ann. Geophysicae, 10, 429-437.

Hide L., Birch L., Morrison V., Shea D.J., White, 1980. "Atmospheric Angular Momentum and changes in the length of the day," Nature, 286, 114-117.

Hide I., Dickey J.O., 1991. "Earth variable rotation." Science, 253, 629-637.

IERS, 1988-1998. IERS Annual Report for 1988-1998, Observatoire de Paris, France,

IERS Conventions (1996), 1996. ed. by D. D. McCarthy, IERS Technical Note 21, Observatoire de Paris, France.

Kołaczek B., Kosek W., 1987. Systematic differences between radiointerferometric and laser series of pole coordinates and short-periodic variations of pole coordinates. Paper presented at the IUGG Symposium V4 "Variations in Earth rotation," Vancouver, Canada. Geophys. Monograph, 82, IUGG, copyright 1994 by the IUGG and AGU, 95-101.

Kolaczek B., 1992. Variations of Short Periodical Oscillations of Polar Motion with Periods ranging from 10-140 days. Report No. 419 Department of Geodetic Science and Surveying. The Ohio State University, Columbus, Ohio 43210-1247, Apr. 1992.

Kolaczek B., 1993. Váriations of Short Periodical Oscillations of Earth Rotation. Proc. of the 156th Symp. of the IAU held in Shanghai, China, Sep. 1519, 1992. Developments in Astrometry and Their Impact on Astrophysics and Geodynamics. I.I. Mueller and B. Kołaczek (eds). Kluwer Academic Publishers. Dordrecht/Boston/New York, 291-296.

Kołaczek B., Kosek W., 1993. Variations of 80-120 days Oscillations of polar motion and Atmospheric Angular Momentum. Proc. 7th International Symposium "Geodesy and Physics of the Earth" IAG Symposium No. 112 , Potsdam, Germany, Oct. 5-10, 1992. H. Montag and Ch. Reigber (eds.), Springer Verlag, 439-442.

Kołaczek B., 1995. Short Period Variations of Earth Rotation. Proc. Journées 1995 "Systèmes de Référence Spatio-Temporels", Warsaw, Poland, Sep. 18-20, 1995. 147-154.

Kołaczek B., Nuzdhina M., Nastula J., Kosek W. 1999. "El Niño impact on atmospheric and geodetic excitation functions" - accepted by Journal Geophysical Research.

Kołaczek B., Nuzhdina M., Nastula J., Kosek W. 1999. "El Niño Impact on Polar Motion," IERS Technical Notes No 26, "The impact of El Niño and other low frequency signals on Earth Rotation and global Earth System parameters" Paris, France. 23-28.

Kosek W., 1987. "Computations of Short Periodical Variations of Pole Coordinates of the MERIT Campaign by the Use of Ormsby Filter and the Maximum Entropy Spectral Analysis with the Optimum Filter Length, Bulletin Géodésique, 61, 109-124. 
Kosek W., 1993. "The Common Short Periodic Oscillations in the Earth's Rate of Rotation, Atmospheric Angular Momentum and Solar Activity," Bulletin Géodésique, 67, 1-9.

Kosek W. 1995. "Time Variable Band Pass Filter Spectra of Real and ComplexValued Polar Motion Series," Artificial Satellites, Planetary Geodesy, 30, 27-43.

Kosek W., Kołaczek B., 1995a. "Irregular Short Period Variations of Polar Motion." Proc. Journées 1995 "Systèmes de Référence Spatio-Temporels", Warsaw, Poland, Sep. 18-20, 1995. 117-120.

Kosek W., Nastula J., Kołaczek B., 1995b. "Variability of polar motion oscillations with periods from 20 to 150 days in 1979-1991." Bulletin Geodesique, 69, 308-319.

Kosek W., Kołaczek B., 1997. "Semi-Chandler and Semiannual Oscillations of Polar Motion." Geophysical Research Letters, 24, 2235-2238.

Kosek W., McCarthy D.D., Luzum B. 1998a. "Possible Improvement of Earth. Orientation Forecast Using Autocovariance Prediction Procedures, Journal of Geodesy, 72, 189-199.

Kosek W., Kołaczek, B., Salstein D., Nastula J., 1998b. "Time variable Spectra of Polar Motion and its Atmospheric Excitation." Paper presented at the AGU Fall Meeting, Symposium G71I, "Polar Motion from Hours to Decades," San Francisco, December 6-10, 1998.

Kosek W., Popiński W., 1999a. "Comparison between the Fourier transform band pass filter and the wavelet transform spectro-temporal analyses on the Earth Rotation Parameters and their excitation functions." Submitted to IERS Technical Notes 28, paper presented at the EGS XXIV General Assembly The Hague, The Netherlands, 19-23 April 1999.

Kosek W., Popiński W., 1999b. "Comparison of the spectro-temporal analysis methods on polar motion and its atmospheric excitation." Accepted to Artificial Satellites, Space Research Centre, PAS, Warsaw, Poland.

Kuehne J., Stuart J., Wilson C.R., 1993. "Atmospheric excitation of nonseasonal polar motion, JGR, 98, 19973-19978.

Morabito D.D., Eubanks T.M., Steppe J.A., 1988. Kalman filtering of Earth rotation changes in the 128th IAU Symposium on The Earth's rotation and reference frames for geodesy and geodynamics. Kluwer Academic, $257-267$.

Nastula J. 1992. "Short periodic variations in the Earth's rotation in the period 1984-1990," Ann. Geophysicae, 10, 441-448.

Nastula J., 1995. "Analysis of the Regional Contributions to the Polar Motion Excitation." Proc. Journées 1995 "Systèmes de Référence SpatioTemporels", Warsaw, Poland, Sep. 18-20, 1995. 155-158.

Nastula J., Kosek W., Kolaczek B., 1997. "Analyses of Zonal Atmospheric Excitation Functions and Their Correlation with Polar Motion Excitation Functions." Annales Geophysicae, 15, EGS 1439-1446.

Nothnagel, A., Nicolson, G.D., Campbell, J. and Schuh, H., 1992. "Radiointerferometric polar motion observations with high temporal resolution," Bulletin Géodésique, 66, 346-354. 
Otnes R.K., Enochson L. 1972. Digital Time Series Analysis, John Wiley and Sons Publishing Company, New York.

Petrov S., Brzeziński A., Gubanov V.S., 1996. "A stochastic model for polar motion with Application to smoothing, prediction and combining." Artificial Satellites, 31,1 (Planetary Geodesy No 26), Space Research Centre, PAS, Warsaw, Poland, 51-70.

Popiński W., Kosek W. 1994. "Wavelet Transform and Its Application for Short Period Earth Rotation Analysis," Artificial Satellites, 29, 75-83.

Popiński W., Kosek W. 1995a. "Discrete Fourier and Wavelet Transforms in Analysis of Earth Rotation Parameters," in Proc. Journées 1995 - Earth Rotation, Reference Systems in Geodynamics and Solar System, 18-20 September, Warsaw, Poland, 121-124.

Popiński W., Kosek W. 1995b. "The Fourier Transform Band Pass Filter and its Application to Polar Motion Analysis," Artificial Satellites, 30, 9-25, Space Research Centre, Warsaw, Poland.

Rosen R.D. and Salstein D.A., 1983. "Variations in Atmospheric Angular Momentum on global and regional scales and the length of day," $J G R, \mathbf{8 8}$, 5451-5470.

Schmitz-Hübsch, H. and Schuh, H. 1999. "Seasonal and Short-Period Fluctuations of Earth Rotation Investigated by Wavelet Analysis." Festschrift for Erik W. Grafarend, Univ. Stuttgart, Technical Reports Dep. of Geodesy and Geoinformatics, Report Nr. 1999.6-2, ed. by F. Krumm and V.S. Schwarze, 421-431.

Schuh, H. 1986. "Analysis of UT1 Determinations by VLBI within Project IRIS." IAU Symposium 128, The Earth's Rotation and Reference Frame for Geodesy and Geodynamics, Coolfont, Oct. 20-24, 1986, ed. by Babcock and Wilkins, D. Reidel, 171-180.

Schuh, H. 1990. "Earth's Rotation Measured by VLBI." Proc. of the 3rd Meeting on Tidal Friction and the Earth's Rotation, Bielefeld, Sep., 1988, ed. by P. Brosche and H. Sündermann, Springer Verlag, 1-12.

Schuh, H., 1999. "The Rotation of the Earth Observed by VLBI." Acta Geod. Geoph. Hungarica, 34, 421-432.

Schuh, H. and O. Titov, 1999, "Short-period variations of the Earth's Rotation Parameters as seen by VLBI." Proc. of the 13th Working Meeting on European VLBI, Viechtach Feb. 1999, ed. by W. Schlüter and H. Haase, BKG report, 172-177.

Titov, O. 1999. "Least squares collocation method (LSCM) for space geodetic data analysis." Proc. of the IAG Symposium "Towards an Integrated Global Geodetic Observing System' (IGGOS), Munich, Oct. 1998, in press, 1999.

Titov, O. and N. Zarraoa, 1997. "OCCAM 3.4 User's Guide." Communications of the Institute for Applied Astronomy (IAA), St. Petersburg, No. 69. 